\title{
Semantic Learning Service Personalized
}

\author{
Yibo Chen \\ Computer School of Wuhan University \\ Wuhan, Hubei, China \\ chenyibo8224@gmail.com \\ Chanle Wu \\ Computer School of Wuhan University \\ National Engineering Research Center for Multimedia Software \\ Wuhan, Hubei, China \\ chanle.wu@gmail.com \\ Xiaojun Guo \\ Computer School of Wuhan University \\ Wuhan, Hubei, China \\ guoxiaojun1026@qq.com \\ Jiyan Wu \\ Computer School of Wuhan University \\ Wuhan, Hubei, China \\ 331588723@qq.com \\ Received 26 October 2011 \\ Accepted 30 January 2012
}

\begin{abstract}
To provide users with more suitable and personalized service, personalization is widely used in various fields. Current e-Learning systems search for learning resources using information search technology, based on the keywords that selected or inputted by the user. Due to lack of semantic analysis for keywords and exploring the user contexts, the system cannot provide a good learning experiment. In this paper, we defined the concept and characteristic of the personalized learning service, and proposed a semantic learning service personalized framework. Moreover, we made full use of semantic technology, using ontologies to represent the learning contents and user profile, mining and utilizing the friendship and membership of the social relationship to construct the user social relationship profile, and improved the collaboration filtering algorithm to recommend personalized learning resources for users. The results of the empirical evaluation show that the approach is effectiveness in augmenting recommendation.
\end{abstract}

Keywords: personalized learning service; semantic information search; ontology; collaboration filtering 


\section{Introduction}

Some of e-Learning researchers divided learning service into application service, education service and common service ${ }^{1}$, each service has different service target. However, there are highly interactive between the various services, clearly separating the functions of the service, easy to separate the internal relations between the services, the integrity of the services also will be lost. At present, personalization technology is increasingly used to the service, to provide users with personalized functions, such as personalized information search, personalized navigation and so on. It is the future development direction of e-learning to combine the personalization with learning service to provide personalized learning service. But there are two problems need to solve:

Firstly, personalizing the retrieval of needed information in an e-learning environment based on context requires intelligent methods for representing and matching both the learning resources and the variety of learning contexts ${ }^{2}$. Some researchers found that semantic technology can provide a representation of the learning content, and the semantic user profiles can form a good representation of the learning context. User profile is the foundation of personalization; personalization is one of the most effective means to provide the exactly resources for users. But lacking of deeply understanding of user and inadequate considering of internal and external factors that impact the user's learning, makes the structure of user profiles is not accurately which increased the difficulty of retrieval of learning resources. So, e-Learning system need to mine the history data, analyze the user's personality, interesting, social relationship, etc. the mainly characteristic which impact learning to enhance the precision of the search through the personalization and semantic technology.

Secondly, we need to define the concepts of learning services, highly integrated service contents and innovative personalized service. Service in the real world means a paid or unpaid activity which usually refers to doing things for others and makes others benefited, not in kind of real object but only in the form of offering living labor to meet the special needs of some others. In the E-learning system, personalized learning service is an activity which mines the user context and understands users' needs to help users to learn faster and better. The services should be imperceptible, inseparability, different in quality and not storability. By constructing a personalized learning service with above characteristics, users can access to learning resources more quickly and easily and be allowed to get more suitable and personalized learning services. Meanwhile, customer satisfaction would be continuously improved.

This paper focuses on the semantic retrieval of learning resources in E-learning, through the highly integrated services blend into the individual elements, proposed personalized learning services based on semantic. This research has the following contributions. (1) The concept of personalized learning services are put forward clearly; (2) A framework of learning services personalized is given; (3) Recommendation of learning resources based on knowledge topic is proposed.

Section II of this paper analyzes the research survey situation. Section III describes the overall learning service personalized framework in detail, and structure of user's profile and personalized learning resources. Section IV demonstrates the superiority of the architecture through specific experiments and analysis. Section $\mathrm{V}$ is a summary and a future prospect of this research.

\section{Related Research}

Personalized search and recommendation are the foundation of the personalized learning service:

In the aspect of personalized search, WebMate ${ }^{3}$ extract the user's query by user profile to satisfy the short-term interesting. The method focuses on collecting the browsing and executing information of the user, but did not announce the specific experimental results. Watson ${ }^{4}$ use the local context to extract user's query, but there is no the user profile. Inquirus ${ }^{5}$ select data source based on the user preference, and extract the user's query, but the system have no user profile, and the user have been required to provide their preference directory. In [6], the author proposed to learn the user profile from the history of 
the user surfing, and re-ranking or filtering the searching outcome which from the search engine based on user profile. Google Personalized Search builds a user profile by means of implicit feedback where the system adapts the results according to the search history of the user. Many systems employ search personalization on the client-side by re-ranking documents that are suggested by an external search engine $^{7,8}$. Since the analysis of the pages in the result list is a time consuming process, these systems often take into account only the top ranked results. Also, only the snippets associated with each page in the search results is considered as opposed to the entire page content. The ODP is the largest and most comprehensive Web directory, which is maintained by a global community of volunteer editors. The ODP taxonomy is used as the basis for various research projects in the area of Web personalization ${ }^{9,10}$. In [11], the author utilize the first three levels of the ODP for learning profiles as bags of words associated with each category. The user's query is mapped into a small set of categories as a means to disambiguate the words in the query, and then The Web search is then conducted based on the user's original query and the set of categories. In [12], the author utilizes the documents stored locally on a desktop PC for personalized query expansion. The query terms are selected for Web search by adapting summarization and natural language processing techniques to extract keywords from locally stored desktop documents. In Persona ${ }^{13}$, the well-known Hyperlink Induced Topic Selection (HITS) algorithm is enhanced with an interactive query scheme utilizing the Web taxonomy provided by the ODP to resolve ${ }^{14}$

In the aspect of personalized recommendation, AVS(Altered Vista System) is one of the first learning resource collaboration filtering system, the aim is to explore how to collect the user's rating for learning resource, and then to propagate them in the form of word-of-mouth recommendations about the qualities of the resources ${ }^{15,16}$. The another learning resource recommender system is RACOFI (Rule Applying Collaborative Filter) ${ }^{17}$, it combines two recommendation approaches by integrating a collaborative filtering engine that works with ratings that users provide for learning resources with an inference rule engine that is mining association rules between the learning resources and using them for recommendation, but the research of the system have not assessed the pedagogical value of the recommender, nor have they reported some evaluation of the system by users. In [18], the author developed QSIA(The Questions Sharing and Interactive Assignments) system for the sharing, assessing and recommendation of the learning resources, it is used in the context of online communities in order to harness the social perspective in learning and to promote collaboration, online recommendation and further formation of learner communities. The system has been implemented and used in the context of several learning situations, such as knowledge sharing among faculty and teaching assistants, high school teachers and among students, but no evaluation results have been reported so far. In learning resource collaboration filtering system, CYCLADES ${ }^{19}$ has proposed an environment where users search, access and evaluate digital resources available in repositories found through the OAI (Open Archives Initiative). In [20], the author developed a recommender system for learning objects that is based on sequencing rules that help users be guided through the concepts of ontology of topics. The rules are fired when gaps in the competencies of the learners are identified, and then appropriate resources are proposed to the learners. In [21], the author proposed a more advanced e-Learning system, open into new learning resources that may be found online, which includes a hybrid recommendation service. Their system is mainly used for storing and sharing research papers and glossary terms among university students and industry practitioners. Resources are tagged according to their content and technical aspects, but learners also provide feedback about them in the form of ratings. Recommendation takes place both by engaging a clustering module and a collaborative filtering module. In [22, 23], the author proposed a learning resource recommender system based on the hybrid recommendation approach, which combines social-based with information-based recommendation techniques. [24] focused on e-learner's emotion category, emphasizing on e-learner's emotional interaction, analyzing interactive Chinese texts, building emotion category database and strategies of database of sentiment adjustment, 
realizing the real-time emotion analysis and recommendation algorithm ${ }^{25}$.

\section{Learning Service Personalized Framework}

Learning service personalized framework is show as figure 1. According to MVC (Model-View-Control) design principle, we divide learning service personalized framework into semantic model layer, control (algorithm) layer, presentation layer. Semantic model layer utilize ontology technology to construct domain ontology, user domain profile ontology and user social relationship ontology. Control (algorithm) layer designs domain ontology clipping algorithm constructs user domain profile ontology; social relationship extracting algorithm to construct user social relationship ontology; personalized recommendation algorithm to personalize the course, semantic search, navigator and recommendation, and provided personalized learning resources for users. Presentation layer includes learner activity, personalized semantic search, personalized semantic navigator, and personalized course centre 4 models. Learner activity model collect the user-machine interaction information; personalized semantic search model present the recommendation list of semantic search outcomes; personalized semantic navigator model provide the personalized knowledge point navigation based on user knowledge background; personalized course centre model custom personalized course according to the context and learning objectives of the user

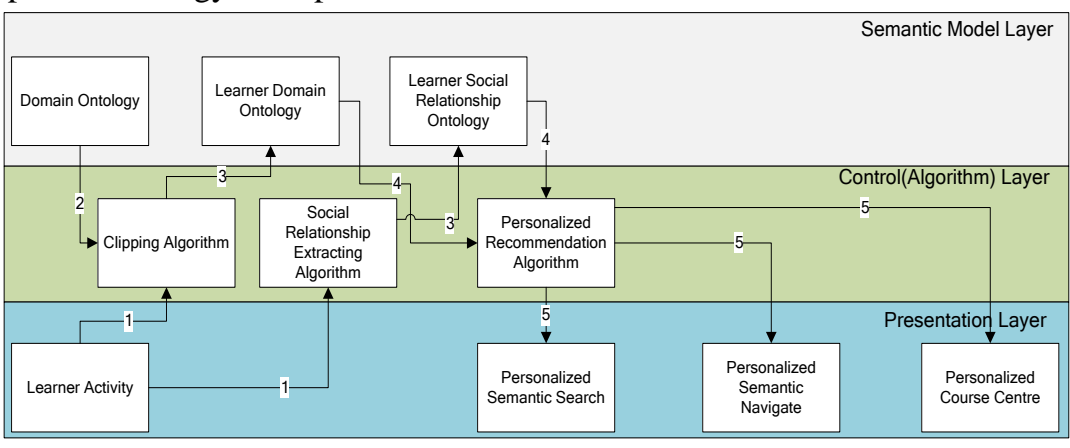

Fig 1. Learning Service Personalized Framework

\subsection{Structure of Domain Semantic}

We use $\mathrm{R}$ to represent the root of the domain which is represented as a tree, and $\mathrm{C}_{\mathrm{i}}$ represent a concept (course) under R. As shown in formula (1).

$$
R=\bigcup_{i=1}^{n} C_{i}
$$

Where $n$ is the number of concepts in the domain. Each concept $C_{i}$ consists of sub-concepts (chapter) $\left(S C_{i j}\right)$,

$$
C_{i}=\bigcup_{j=1}^{n 1} S C_{i j}
$$

Where $n 1$ is the number of sub-concepts. Each concept $S C_{i j}$ consists of meta-sub-concepts (section) $\left(M S C_{i j k}\right)$,

$$
S C_{i j}=\cup_{k=1}^{n 2} M S C_{i j k}
$$

Where $\mathrm{n} 2$ is the number of meta-sub-concepts. Each meta-sub-concepts $M S C_{i j k}$ consists of meta-meta-sub-concepts (Knowledge Point) which can be children, $\left(M M S C_{i j k l}\right)$, i.e.,

$$
M S C_{i j k}=\bigcup_{l=1}^{n 3} M M S C_{i j k l}
$$

$\mathrm{MMSC}_{\mathrm{ijkl}}$ is high-dimensional variable. For instance, memory is a section which is a meta-sub-concept $M S C_{i j k}$. Memory also can be divided into RAM, ROM, etc., then the RAM and ROM is meta-meta-sub-concepts $\mathrm{MMSC}_{\mathrm{ijk}}$.

We encoded the above semantic information into tree structured domain ontology in OWL, based on the hierarchy of the course. The root concepts are the special domain, while the sub-concepts are the courses, chapter, section or knowledge point. Each child node holds the following information: <node name, parent node, child node, visit number>。

\subsection{Constructing User Profile}

\subsubsection{User Domain Profile}

Because the log of the user access activity shows the visited resources, we design a bottom-up clipping algorithm which extract semantic user concepts the user interested in, and then build the semantic user domain 
profiles by extracting the user interests from the semantic domain. Every user has a dynamic semantic representation. We collect the user's activities over a period of time to form an initial user domain profile, Resources $\left(U_{i}\right)=\bigcup_{m=1}^{n 4} \operatorname{Res}_{i j k m}$ indicates the visited resources by the $i^{\text {th }}$ user. Starting from the leaves, the clipping algorithm searches for every resources that the user visited in the domain semantic structure, and then increases the visit number (initialized with 0 ) of each visited node up to the root. After statistic finished, the clipping algorithm only keeps the concepts, sub-concepts, meta-sub-concepts and meta-meta-sub-concepts related to the user interests with their weighted interests which are the number of visits. Algorithm 1 shows the clipping steps.

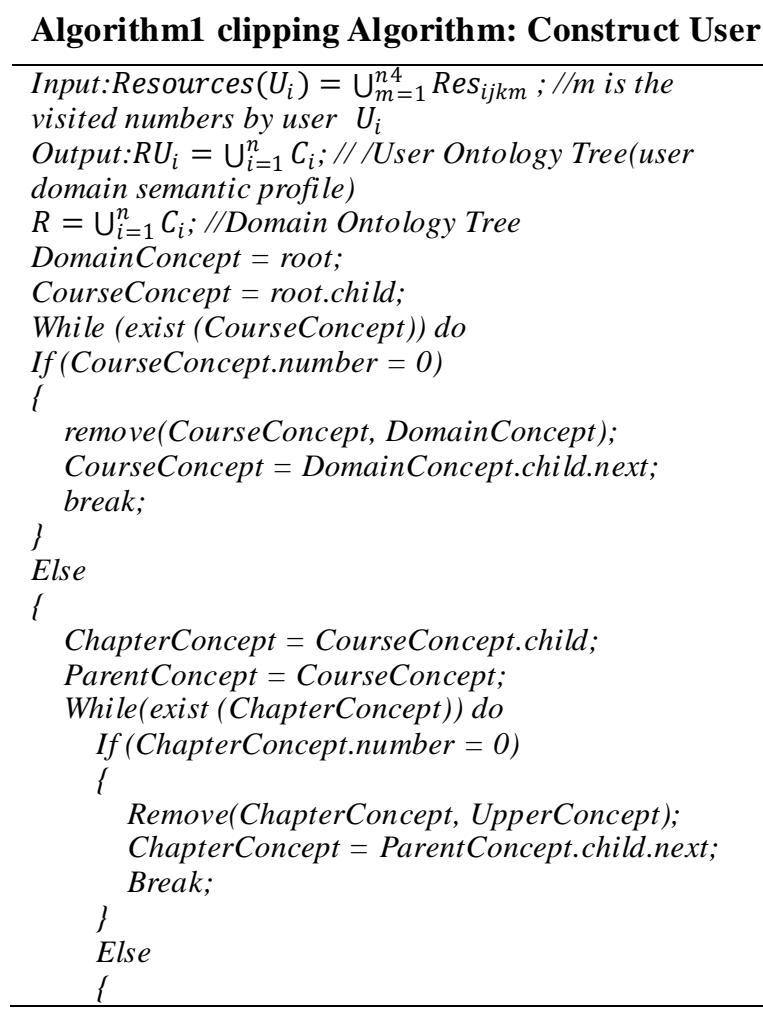

\subsubsection{User social relationship profile}

Friend recommendation and group recommendation which are important parts in the real world usually have higher feasibility, therefore, these recommendations has an important impact on the quality of personalized learning service. The experimental system of this project provides getFriends() and getGroups() interfaces. User's friends list and a list of their respective study groups are available through the two interfaces. The specific information collection methods and implementations are not the keys of this article; therefore, this paper has not a detailed description about that. Figure 2 shows a user social relationship profile ontology, which includes the user and the user's friends information and

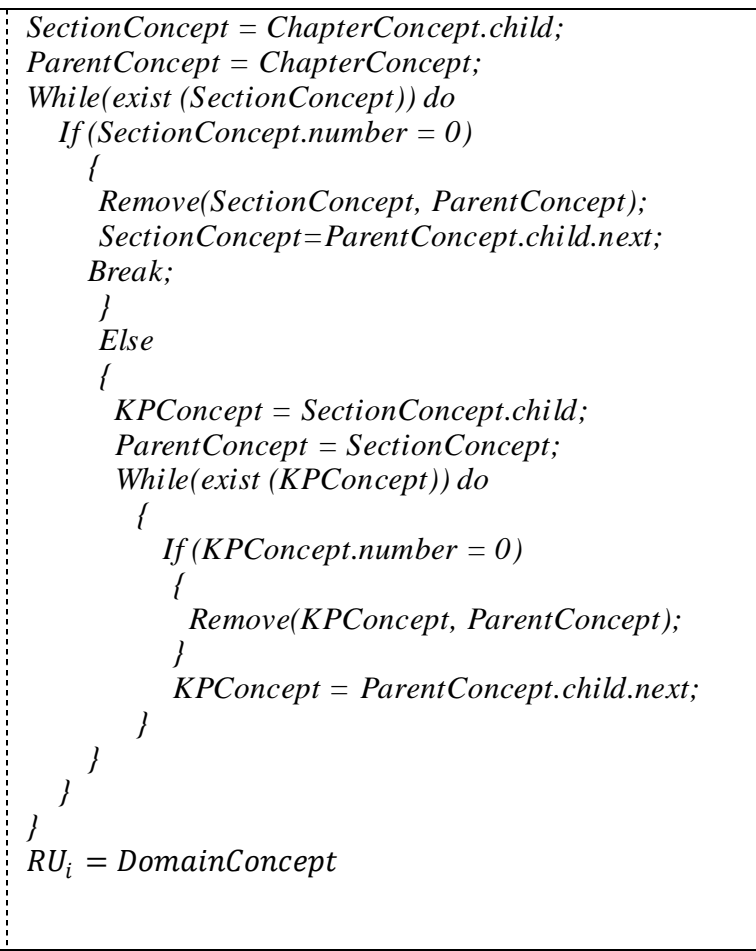

information of the study group that user joined in. algorithm 2 indicates the construction process of the user social relationship profile ontology, and the output of the algorithm is the user social relationship profile ontology.

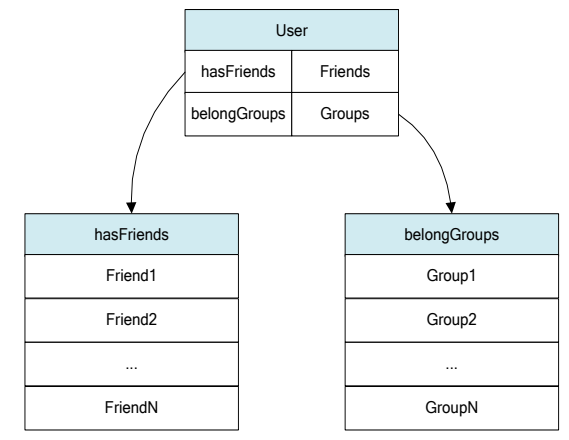

Fig 2. User Social Relationship Ontology 


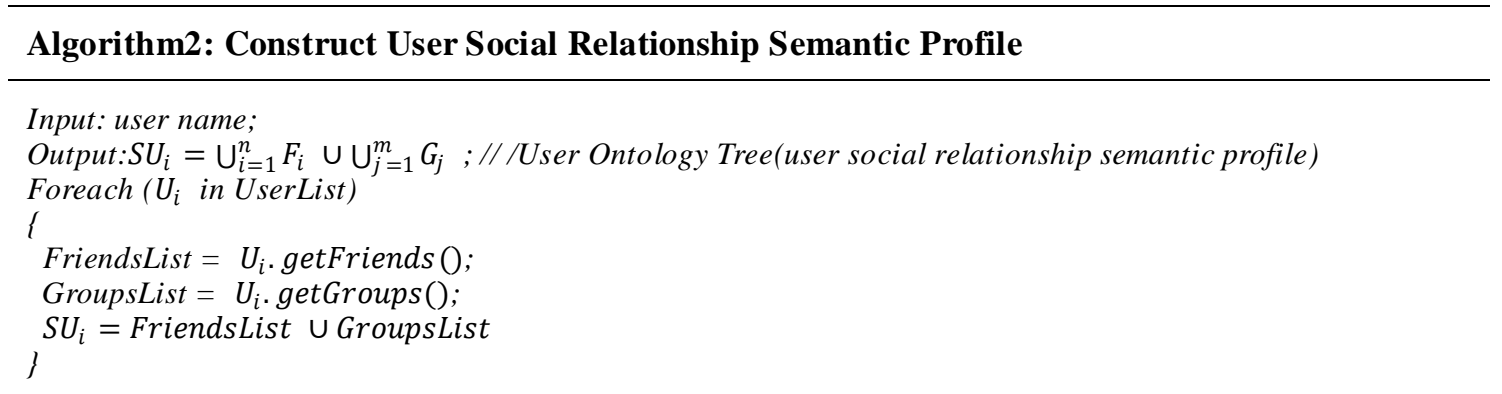

\subsection{Learning Resource Personalized}

In the process of learning resource personalized, firstly, filtering the contents the user uninterested through the user domain profile ontology, and sorting the return list according to the interesting weight. In order to achieve better recommendation effecting, we utilize collaboration filtering recommendation approach to re-rank the return list according to the user similarity, it is computed based on user-resource rating matrix, user-friend matrix and user-group matrix.

To filter the resources user uninterested, we use a term vector $\overrightarrow{r e s}=\left\langle w_{1}, w_{2}, \cdots, w_{n}\right\rangle$ to represent each resources based on VSM(Vector Space Model) [26, 27], $w_{i}=t f_{i} * \log N / n_{i}$ is the term weight for term $i, t f_{i}$ indicates the term frequency, and $\log N / n_{i}$ is the term's Inverse Document Frequency if this term occurs in $n_{i}$ resource. When a user searches for resources using a specific query $q(q$ is inputted keyword, navigation title or concept which extracted from the user domain profile ontology by personalized recommendation), Pearson similarity measure is used to retrieve the most similar resources that contain the terms in the query. The results have been filtered based on the semantic relationship between these resources and the user's semantic domain profile.

The above filtering process only judge whether the user is satisfied with the search results. In order to provide more accurate resource, re-ranking the recommendation list is needed. The experiment system design a rating mechanism for learning resource, the user can rate learning resources he/she used by the rating mechanism, rating scope is 1 to 5,5 represent very satisfaction, 1 represent very no satisfaction. In the learning process of the user, information collecting model will collect the rating for every users. At the time of collaboration filtering, we mainly take into account of friendship and membership two types of explicit social relationships, and proposed an approach to fuse them with the user-resource rating matrix. User similarity computation is crucial to collaborative recommenders; a more accurate user similarity always leads to better recommendation results. In this paper, our proposed approach is to leverage the two social relationships to strengthen the user similarity calculation process by combining the user similarity from friendship and/or membership with similarity from rating matrix in a weighted approach.

Classical user similarity computing methods mainly include Vector Cosine Angle (formula 5) and Pearson Correlation Coefficient (formula 6).

$$
\begin{aligned}
& \operatorname{Sim}(x, y)=\frac{\sum_{s \in S_{x y}} r_{x, s} \cdot r_{y, s}}{\sqrt{\sum_{s \in S_{x y}} r_{x, s}^{2} \sum_{s \in S_{x y}} r_{y, s}^{2}}} \\
& \operatorname{Sim}(x, y)=\frac{\sum_{s \in S_{x y}}\left(r_{x, s}-\overline{r_{x}}\right)\left(r_{y, s}-\overline{r_{y}}\right)}{\sqrt{\sum_{s \in S_{x y}}\left(r_{x, s}-\overline{r_{x}}\right)^{2} \sum_{s \in S_{x y}}\left(r_{y, s}-\overline{r_{y}}\right)^{2}}}
\end{aligned}
$$

$S_{x}, S_{y}$ is a rating set of user $x$ and user y respectively. $S_{x y}$ represent the intersection set between $S_{x}$ and $S_{y}$. $\overline{\mathrm{r}}_{\mathrm{x}}, \overline{\mathrm{r}}_{\mathrm{y}}$ is the average rating of user $\mathrm{x}, \mathrm{y}$.

Classical computing method are mainly based on user-resource rating matrix UI (our user-resource matrix only include the filtered resource). In this paper, we collect the social relationship of the users to construct user-friend and user-group matrix UF, UG respectively. $U I_{n, m}$ (User-Item Matrix), $\mathrm{n}$ is the number of user, $m$ is the number of item, the value of the cell is the rating of user to item. A column from this matrix is referred to as an item's user profile, containing a distribution of the most prominent item users. $U F_{n, l}$ (User-Friends Matrix), 1 is the number of friend. $U G_{n, k}$ (User-Group matrix), $\mathrm{k}$ is the number of group. We utilize Pearson similarity to compute the similarity $\operatorname{Sim}_{U I}(x, y), \operatorname{Sim}_{U F}(x, y), \operatorname{Sim}_{U G}(x, y)$ and to assign different weight respectively. We compute the user similarity and the predictive rating according to 
formula (7) and (8) respectively. At last, we re-rank the recommendation list according to the product between

$$
\begin{aligned}
& \operatorname{Sim}(x, y)=\alpha \operatorname{Sim}_{U I}(x, y)+(1-\alpha)\left(\beta \operatorname{Sim}_{U F}(x, y)+(1-\beta) \operatorname{Sim}_{U G}(x, y)\right) \\
& r_{x, i}=\bar{r}_{x}+k \sum_{\bar{x} \in X} \operatorname{Sim}(x, \bar{x}) \cdot\left(r_{\bar{x}, i}-\bar{r}_{x}\right) \\
& k=1 / \sum_{\bar{x} \in X} \operatorname{Sim}(x, \bar{x})
\end{aligned}
$$

the predictive rating and the interesting degree.

$\mathrm{X}$ represent the most similarity top- $\mathrm{N}$ users set. The value of similarity located in $[0,1]$. The parameter $\alpha$ is used to adjust the weight of the user's social relationship and $\operatorname{Sim}_{U I}(x, y)$, the bigger the $\alpha$ is, the rating matrix plays a more important role in the combined similarity. $\beta$ is used to adjust the weight of $\operatorname{Sim}_{U F}(x, y)$ and $\operatorname{Sim}_{U G}(x, y)$, the bigger the $\beta$ is, the

friendship plays a more important role. The value of $\alpha$ and $\beta$ located in $[0,1]$. We can set the different value of $\alpha$ and $\beta$ according to the different situation. For example, if UI and UG is very sparsity, then set the $\alpha$ little and the $\beta$ big. Otherwise, through experiment, the user achieve the highest satisfaction for the recommendation when $\alpha=0.6$ and $\beta=0.7$.

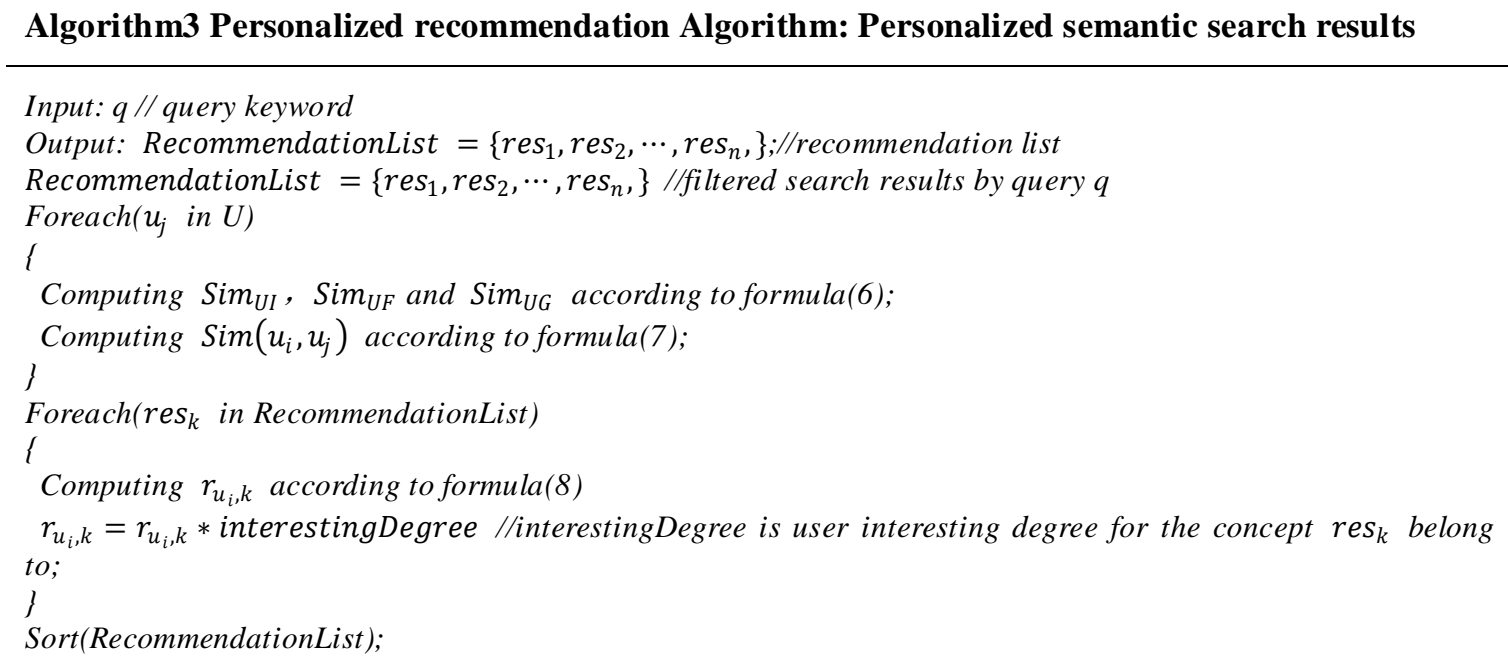

\section{Experiment and Analysis}

\subsection{Experiment environment}

The experiment system has been established in our laboratory Research of E-learning platform (National High Technology Research and Development Program of China (863)). The old platform searches the learning resource based on the keywords, it has the collaboration recommendation ability, but does not have the semantic inference ability and the ontology user profile, also not consider the user's social relationship, such as friend relationship and group relationship. The experiment system has 10 courses in computer field, and a total of 3806 learning resources.

\subsection{Measurement}

We used Recall and Precision to measure the effectiveness of the proposed framework. The definition is as shown in formula 10 and formula 11 . The experiment base dataset collected from the old system. The recall and precision of the old system are as shown in figure 3 and figure 4 . We compared the search result provided by the old system with the result of personalized semantic search engine the experimental system provides.

$$
\begin{aligned}
& \text { Recall }=\frac{\text { Number of recommended items that match with future views }}{\text { Total number of items in future views }} \\
& \text { Precision }=\frac{\text { Number of recommended items that matc } h \text { with future views }}{\text { Total number of recommended items }}
\end{aligned}
$$




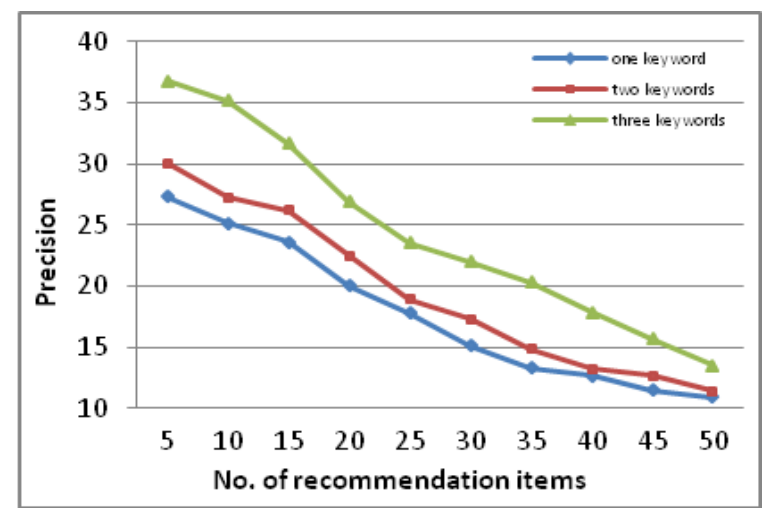

Fig 3. The value of precision

\subsection{Experiment process}

We selected 10 users and create the user domain profile and the user social relationship profile for each user using Algorithm 1 and Algorithm 2 respectively. A total

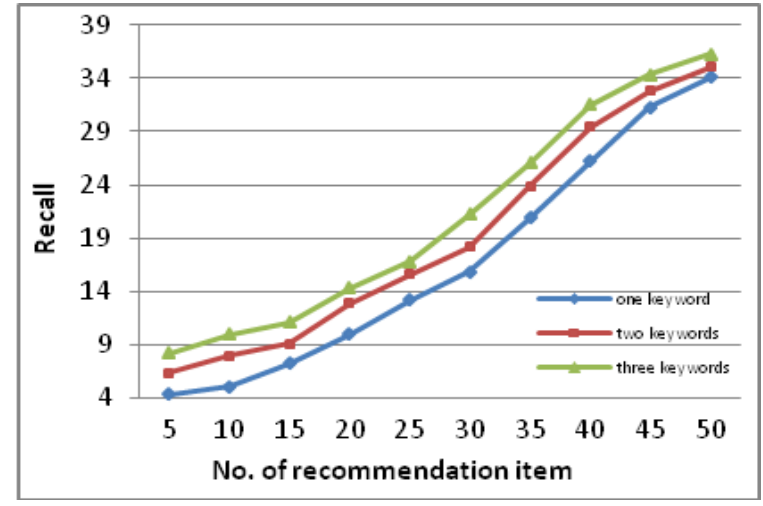

Fig 4. The value of recall

of 1512 resources represented the user profiles, with the size of each profile varying from one learner to another, as shown in Table 1. In the experiment, the length of the query keyword is 1,2 and 3 respectively.

Table 1. The number of resources in the user profile

\begin{tabular}{|c|c|c|}
\hline User Name & Resource Number & Course \\
\hline User 1 & 96 & Computer System and Interface Technology \\
\hline User 2 & 80 & Computer Network \\
\hline User 3 & 167 & $\begin{array}{l}\text { Computer System and Interface Technology } \\
\mathrm{C}++\end{array}$ \\
\hline User 4 & 223 & $\begin{array}{l}\text { Computer Network } \\
\text { Principles of Compiler } \\
\text { Operating System }\end{array}$ \\
\hline User 5 & 139 & principles of computer organization \\
\hline User 6 & 374 & Object-oriented Program \\
\hline User 7 & 106 & Data Mining \\
\hline User 8 & 78 & Nature Language Process \\
\hline User 9 & 117 & Grid Computing \\
\hline User 10 & 132 & $\begin{array}{l}\text { Data Mining } \\
\text { Nature Language Process }\end{array}$ \\
\hline
\end{tabular}

\subsection{Experiment result}

achieved 5\%-25\% compares to the old system; Figure

Experiment result as shown in Figure 5 and Figure 6.

Figure 5 shows that the improvement of the precision

\section{Conclusion and Future Work}

E-Learning is a very challenging research field; appropriate and personalized learning services can help the user to get knowledge in an even better fashion. The paper analyzes the research status, the concept of personalized learning services are put forward clearly and a general learning services personalized framework is proposed. The framework made full use of semantic technology, using ontology to represent the learning

6 shows that the improvement of the recall achieved $5 \%-40 \%$.

contents and user profile, mining and utilizing the friendship and membership of the social relationship to construct the user social relationship profile, and improved the collaboration filtering algorithm to recommend personalized learning resources for users. Experiments showed that the proposed framework is effective.

In this work, otherwise, we utilized the friendship and membership of the social relationship to improve the standard of the personalization in this paper. We will 
deeply research the factors of impacting the personalized learning in the future, such as the timeliness of the learning resource, label technology and user's emotion and so on.

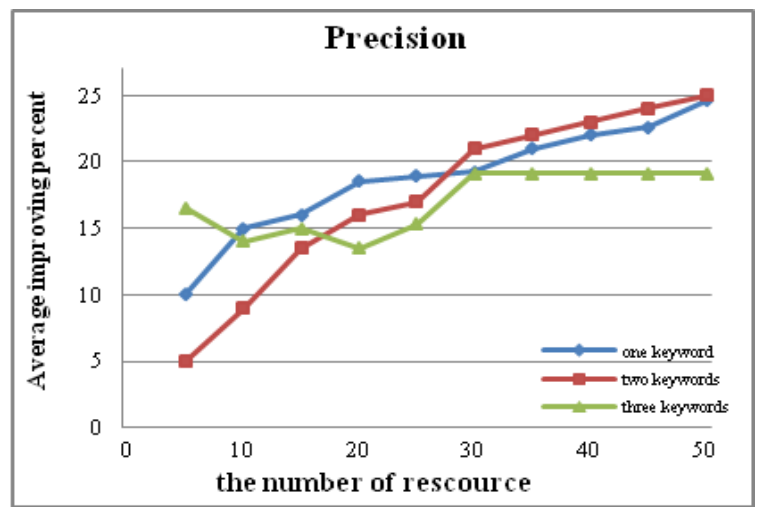

Fig 5. Precision comparison

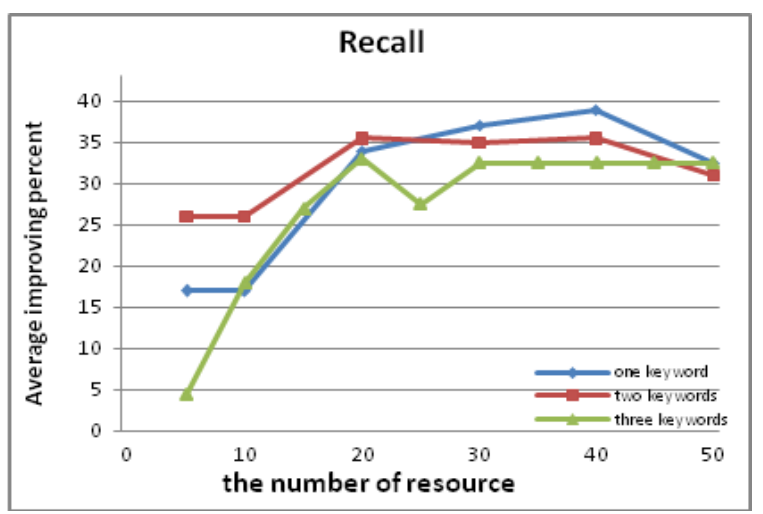

Fig 6. Recall comparison

\section{Acknowledgement}

This work was supported in the National Natural Science Foundation of China under Grant No. 60672051. The Fundamental Research Funds for the Central Universities (3105005). Wuhan science and technology plan projects (201010621209).

\section{Reference}

1. Dagger, D. O'Connor, A. Lawless, S. Walsh, E. Wade, V.P Service-Oriented E-Learning Platforms From Monolithic Systems to Flexible Services, Internet Computing. 11(3)(2007):28-35.

2. Leyla Zhuhadar, Olfa Nasraoui. Semantic Information Retrieval for Personalized E-Learning. 20th IEEE
International Conference on Tools with Artificial Intelligence (vol. 1, 2008), pp.364-368.

3. L. Chen, K. Sycara. WebMate: A Personal Agent for Browsing and Searching. (Autonomous Agents and Multi Agent Systems, 1998).

4. J. Budzik, J. K. Hammond. Watson: Anticipating and contextualizing information needs, in Proceedings of the Sixty-second Annual Meeting of the American Society for Information Science, 1999.

5. E. Glover, G. Flake, S. Lawrence, W. Birmingham, A. Kruger, C. Giles, D. Pennock. Improving Category Specific Web Search by Learning Query Modifications. (SAINT, 2001).

6. 6. A. Pretschner, S. Gauch. Ontology based personalized search. (ICTAI, 1999).

7. M. Speretta, S. Gauch. Personalized search based on user search histories. In Proceedings of the 2005 IEEE/WIC/ACM International Conference on Web Intelligence, WI 2005 (Compigne, France, September 2005), pp. 622-628.

8. A. Micarelli, F. Sciarrone. Anatomy and empirical evaluation of an adaptive web-based information filtering system, User Modeling and User-Adapted Interaction. 14(2-3)(2004)159-200.

9. P. A. Chirita, W. Nejdl, R. Paiu, C. Kohlschutter. Using odp metadata to personalize search, in Proceedings of the 28th Annual International ACM SIGIR Conference on Research and Development in Information Retrieval, SIGIR 2005 (Salvador, Brazil, August 2005), pp. 178-185.

10. C. Ziegler, K. Simon, G. Lausen. Automatic computation of semantic proximity using taxonomic knowledge, in Proceedings of the 15th ACM International Conference on Information and Knowledge Management, CIKM 2006 (Arlington, VA, November 2006), pp. 465-474.

11. F. Liu, C. Yu, W. Meng. Personalized web search for improving retrieval effectiveness, IEEE Transactions on Knowledge and Data Engineering. 16(1)(2004) 28-40.

12. P. Chirita, C. Firan, W. Nejdl. Summarizing local context to personalize global web search, in Proceedings of the 15th ACM International Conference on Information and Knowledge Management, CIKM 2006 (Arlington, VA, November 2006), pp. 287-296.

13. F. Tanudjaja and L. Mui. Persona: A contextualized and personalized web search. In Proceedings of the 35th Annual Hawaii International Conference on System Sciences, HICSS 2002 (Big Island, Hawaii, January 2002), p. 67.

14. A. Sieg, B. Mobasher, R. Burke. Learning Ontology-Based User Profiles: A Semantic Approach to Personalized Web Search, IEEE Intelligent Informatics Bulletin. (2007)

15. Recker M, Walker A. Supporting 'word-of-mouth' social networks via collaborative information filtering, 
Journal of Interactive Learning Research. 14(2003)79-98.

16. Walker A., Recker M., Lawless K, Wiley D. Collaborative information filtering: a review and an educational application, International Journal of Artificial Intelligence in Education. 14(2004)1-26.

17. Lemire D. Scale and translation invariant collaborative filtering systems, Information Retrieval. 8(2005)129-150.

18. Rafaeli S, Dan-Gur Y, Barak M. Social recommender systems: recommendations in support of e-learning, Journal of Distance Education Technologies. 3(2005)29-45.

19. Avancini H, Straccia U. User recommendation for collaborative and personalized digital archives, International Journal of Web Based Communities. 1(2005)163-175.

20. Shen L, Shen R. Learning content recommendation service based on simple sequencing specification, in Proc. of the ICWL 2004, Vol. 3143, 2004, pp. 363-370.

21. Tang T.Y, McCalla G.I. Smart recommendation for an evolving e-learning system: architecture and experiment, International Journal on E-Learning. 4(2005)105-129.

22. Drachsler H, Hummel H.G.K, Koper R. Recommendations for learners are different: applying memory-based recommender system techniques to lifelong learning, in Proc. of the Workshop on Social Information Retrieval in Technology Enhanced Learning (SIRTEL 2007), 2007, pp.18-26.

23. Drachsler H, Hummel H.G.K, Van den Berg B, Eshuis J, Berlanga A, Nadolski R, Waterink W, Boers N, Koper R. Effects of the ISIS Recommender System for navigation support in self-organized learning networks, Journal of Educational Technology \& Society. 12(2009)122-135.

24. Feng Tian, Qinghua Zheng, Ruomeng Zhao, Tonghao Chen, Xinyan Jia. Can e-learner's emotion be recognized from interactive Chinese texts, in proceedings of the 2009 13th International Conference on Computer Supported Cooperative Work in Design (CSCWD 2009), pp.546-551.

25. N. Manouselis, R. Vuorikari, F. Van Assche. Collaborative recommendation of e-learning resources: an experimental investigation, Journal of Computer Assisted Learning. 26(2010)227-242

26. Salton, G. The SMART retrieval system: experiments in automatic document processing. (New Jersey: Prentice Hall, 1971)

27. Salton, G. Automatic text processing. (MA: Addison-Wesley Publishing Co, 1988) 